\title{
Risk factors and clinical phenotypes of Beijing genotype strains in tuberculosis patients in China
}

\author{
Yu Pang ${ }^{\dagger}$, Yuanyuan Song $^{\dagger}$, Hui Xia ${ }^{\dagger}$, Yang Zhou, Bing Zhao and Yanlin Zhao
}

\begin{abstract}
Background: Beijing genotype strains are the most predominant strains in China. The aim of this study was to explore risk factors and clinical phenotypes associated with infection with Beijing genotype strains among tuberculosis patients in China.

Methods: Using data and strains derived from the first Chinese national drug resistance base-line survey, we performed a statistical analysis of the relationship between different genotypes, demographic characteristics and clinical phenotypes.

Result: Of patients infected with the 3634 strains for which detailed information was available, we found that people in young age groups [aged under 25 years, OR (95\% Cl): 1.30(1.03-1.62)], urban people [OR (95\% Cl): 1.18 (0.47-0.94)], or of Hui ethnicity [OR (95\% Cl): 1.96 (1.10-3.50)] or those needing retreatment [OR (95\% Cl): 1.22 (1.03-1.43)] were more likely to be infected with Beijing genotype strains compared with patients who were rural, or of Han ethnicity or those with new TB cases. In contrast, Uyghur [OR (95\% Cl): 0.45 (0.30-0.67)], or Zhuang ethnicities [OR ( $95 \% \mathrm{Cl}): 0.30(0.19-0.48)]$, presented lower than average risk in infections with the Beijing genotype strain. In addition, a higher proportion of patients with hemoptysis [OR (95\% Cl): 0.81 (0.69-0.94)] and chest pain [OR (95\% Cl): 0.79 (0.69-0.91)] were infected with non-Beijing genotype strains than with Beijing genotype strains.

Conclusions: In China, young age group, urban people, Hui ethnicity and the earlier treated patients are all high risk factors for infection with Beijing genotype strains, while Uyghur and Zhuang ethnicity are lower than average risk factors for infection. The high rate of chest symptoms occurring in non-Beijing genotype infected patients indicates that more attention should be paid to basic research on non-Beijing genotype strains.
\end{abstract}

\section{Background}

Despite global efforts to combat tuberculosis (TB), TB is the most widespread infectious disease in the world [1]. According to a World Health Organization (WHO) report, TB causes nearly 50 to 100 million infections, 9.4 million new cases and 1.3 million deaths every year $[2,3]$, and the situation in developing countries is especially serious. China has the second-highest TB-burden globally, behind only India [3], and data from the $5^{\text {th }}$ national TB epidemiological survey conducted in 2010 indicate that TB has an estimated incidence of 459 cases per 100,000 [95\% confidence interval (CI): 433-484] in China, with 4.99 million prevalent TB cases (95\% CI: 4.71-5.27), including 1.19 million pulmonary, bacterio-

\footnotetext{
* Correspondence: zhaoyanlin@chinatb.org

${ }^{\dagger}$ Equal contributors

National Center for Tuberculosis Control and Prevention, Chinese Center for Disease Control and Prevention, Beijing 102206, China
}

logically confirmed cases (95\% CI: 1.03-1.35) [4]. Although both incidence and mortality rate decreased from 2000 to 2010, TB is still a major public health problem in China due to lack of health-care facilities and other social constraints.

Among the various $M$. tuberculosis genotypes, the Beijing genotype is one of the most prevalent spoligotypes worldwide, especially in eastern Asia, South Africa, and northern Eurasia [5-9], and has been responsible for outbreaks of multidrug-resistant tuberculosis in several parts of the world [10-12]. As shown in many albeit not all settings, various characteristics of the Beijing genotype, including its higher virulence, ability to escape from BCG vaccine-induced protection, and significant association with multidrug resistance, may be responsible for its rapid dissemination [7,13-17]. To date, few studies have focused on the risk factors and clinical phenotypes associated with $M$. tuberculosis Beijing genotype
C Biomed Central (c) 2012 Pang et al.; licensee BioMed Central Ltd. This is an Open Access article distributed under the terms of the Creative Commons Attribution License (http://creativecommons.org/licenses/by/2.0), which permits unrestricted use, distribution, and reproduction in any medium, provided the original work is properly cited. 
strains in patients with tuberculosis. Young age $(0-25$ years of age) and HIV co-infection are reported to be risk factors for Beijing genotype infection in different settings $[18,19]$. Parwati et al. have shown that, in Indonesia, TB treatment is less effective against Beijing genotype strains even when drug resistance is not involved [20]. Hence, an investigation of the risk factors and clinical phenotypes associated with the Beijing genotype in regions where the Beijing genotype is the endemically prevalent strain may have important implications for Tuberculosis Control Programs. In another study [21], we spoligotyped the strains isolated from the first national drug resistance base-line survey of China [22]; $62.2 \%$ of these strains were identified as having the Beijing genotype. Here, we explore the risk factors and clinical phenotypes of Beijing genotype strains among tuberculosis patients in China, using spoligotyping data and patient information derived from the above study.

\section{Methods}

\section{Patient information and bacterial strains}

The protocols applied in this study were approved by the Ethics Committee of the Chinese Center for Disease Control and Prevention. All patients enrolled in the surveillance provided written informed consent, and personal information, including demographic chrematistics and clinical phenotypes.

A total of 4017 clinical strains of tuberculosis, isolated from the base-line surveillance of tuberculosis in 2007 [22], were collected from 31 provinces covered by the National TB Control Program (NTP) in China. The number of patients enrolled in surveillance was proportionally based on patient numbers in different provinces. Cultures from different survey points were transferred to the National Tuberculosis Reference Laboratory by air. Bacterial strains were then recovered on L-J medium for 4 weeks at $37^{\circ} \mathrm{C}$. Genomic DNA was extracted from fresh cultures as reported previously [23]. In brief, spoligotyping was performed with an available spoligotyping kit (Isogen Bioscience BV) according to the manufacturer's protocol [21]. Beijing genotype strains were defined as those which hybridized with all of the last nine spacer oligonucleotides (spacers 35 to 43 ) and none of the first 34 spacer oligonucleotides, while Beijing-like genotype strains were those that hybridized to only some of the last nine spacers.

\section{Data analysis}

Differences in demographic and clinical characteristics between patients infected with M. tuberculosis Beijing genotype strains and patients infected with non-Beijing genotype strains were analyzed by the chi-square test and expressed as odds ratios (ORs) with 95\% confidence intervals (CIs). The multivariate analysis was conducted using the surveylogistic process. All calculations were performed in SPSS 11.5 (SPSS Inc., USA), and the level of significance of univariate analysis was 0.05 , and that of multivariate analysis was 0.10 . The corrected R2 was used as the standard for model screening.

\section{Results}

M. tuberculosis strains were isolated from 3634 patients who had positive sputum smear results and detailed demographic and clinical information. Spoligotyping analysis indicated that 2290 (63\%) of these strains belonged to the Beijing genotype. A classification of patients infected with a Beijing genotype strain, stratified according to gender, age, ethnicity, occupation, education and income, is shown in Table 1. Overall, the percentage of men infected with a Beijing genotype strain was similar to that of women, and education level, income, family size and average family income had no influence on the prevalence of infection with Beijing genotype strains in China. We found that the prevalence of infection with Beijing genotype strains was higher among young patients [aged under 25 years OR (95\% CI): 1.30(1.03-1.62)].

Statistical analysis revealed that the percentage of people infected with Beijing genotype strains differed according to ethnicity. Compared with the percentage of Beijing genotype strains isolated from people of Han ethnicity, the percentage of strains isolated from people of Hui ethnicity [OR (95\% CI): 1.96 (1.10-3.50)] was higher, while the percentage of strains isolated from people of Uyghur [OR (95\% CI): 0.45 (0.30-0.67)], Zhuang [OR (95\% CI): $0.30 \quad(0.19-0.48)]$ and other Chinese ethnicities [OR (95\% CI): 0.67(0.48-0.93)] was significantly lower. There were no statistically significant differences in the rate of infection with Beijing genotype strains between Han and Tibetan ethnicities.

Occupations associated with infection with Beijing or non-Beijing strains were compared. As shown in Table 1, there were significantly more urban people [OR $(95 \%$ CI): 1.18 (0.47-0.94)] among tuberculosis patients infected with Beijing genotype strains, indicating that urban people are a risk factor for infection with strains of the Beijing genotype. We also performed the multivariate analysis towards the distribution of people infected with Beijing genotype strains based on the univariate analysis. As shown in Table 2, the analysis data revealed that young age group, urban people and Hui ethnicity are all high risk factors for infection with Beijing genotype strains, while Uyghur and Zhuang ethnicity are lower than average risk factors for infection, similar to univariate analysis.

Clinical phenotypes of patients infected with Beijing genotype or non-Beijing genotype strains are shown in Table 3. The percentage of cases requiring retreatment 
Table 1 Demographic characteristics of tuberculosis patients according to Mycobacterium tuberculosis genotype

\begin{tabular}{|c|c|c|c|c|}
\hline \multirow[t]{3}{*}{ Characteristic } & \multirow{2}{*}{$\frac{\text { Beijing genotype }}{(2290)}$} & \multirow{2}{*}{$\frac{\text { Non-Beijing genotypes }}{(1344)}$} & \multirow{3}{*}{$\frac{\text { Odds ratios }}{(95 \% \mathrm{Cl})}$} & \multirow{3}{*}{$\begin{array}{c}P \\
\text { value }\end{array}$} \\
\hline & & & & \\
\hline & $\mathrm{N}(\%)$ & N (\%) & & \\
\hline \multicolumn{5}{|l|}{ Sex } \\
\hline Male & $1637(71.5 \%)$ & $964(71.7 \%)$ & 1.00 & - \\
\hline Female & $653(28.5 \%)$ & $380(28.3 \%)$ & $1.01(0.87-1.18)$ & 0.909 \\
\hline \multicolumn{5}{|l|}{ Age group (years) } \\
\hline$<25$ & $403(17.6 \%)$ & $202(15.0 \%)$ & $1.30(1.03-1.62)$ & 0.025 \\
\hline $25-34$ & $353(15.4 \%)$ & $210(15.6 \%)$ & $1.09(0.87-1.37)$ & 0.452 \\
\hline $35-44$ & $391(17.1 \%)$ & $233(17.3 \%)$ & $1.09(0.87-1.36)$ & 0.464 \\
\hline $45-54$ & $393(17.2 \%)$ & 208(15.5\%) & $1.23(0.98-1.54)$ & 0.076 \\
\hline $55-64$ & $316(13.8 \%)$ & $209(15.6 \%)$ & $0.98(0.78-1.24)$ & 0.906 \\
\hline$>65$ & $434(18.9 \%)$ & $282(21.0 \%)$ & 1.00 & - \\
\hline \multicolumn{5}{|l|}{ Ethnicity } \\
\hline Han & $2073(90.5 \%)$ & $1151(85.6 \%)$ & 1.00 & - \\
\hline Hui & $53(2.3 \%)$ & $15(1.1 \%)$ & $1.96(1.10-3.50)$ & 0.021 \\
\hline Tibetan & 12(0.5\%) & $5(0.4 \%)$ & $1.33(0.47-3.79)$ & 0.800 \\
\hline Uyghur & $46(2.0 \%)$ & $57(4.2 \%)$ & $0.45(0.30-0.67)$ & $<0.001$ \\
\hline Zhuang & $27(1.2 \%)$ & $50(3.7 \%)$ & $0.30(0.19-0.48)$ & $<0.001$ \\
\hline Others & 79(3.4\%) & $66(4.9 \%)$ & $0.67(0.48-0.93)$ & 0.017 \\
\hline \multicolumn{5}{|l|}{ Occupation } \\
\hline Rural & $1470(64.2 \%)$ & $912(67.9 \%)$ & 1.00 & - \\
\hline Urban & $820(35.8 \%)$ & $432(32.1 \%)$ & $1.18(1.02-1.36)$ & 0.025 \\
\hline \multicolumn{5}{|l|}{ Education Level } \\
\hline Illiterate & $465(20.3 \%)$ & $274(20.4 \%)$ & 1.00 & - \\
\hline Primary school & $686(30.0 \%)$ & 448(33.3\%) & $0.90(0.75-1.09)$ & 0.308 \\
\hline Middle school & 719(31.4\%) & $432(32.1 \%)$ & $0.98(0.81-1.19)$ & 0.846 \\
\hline High school & $319(13.9 \%)$ & $148(11.0 \%)$ & $1.27(0.99-1.62)$ & 0.063 \\
\hline University & $101(4.4 \%)$ & $41(3.1 \%)$ & $1.45(0.98-2.15)$ & 0.069 \\
\hline \multicolumn{5}{|l|}{ Income (USD) } \\
\hline$<348$ & $446(19.5 \%)$ & $244(18.2 \%)$ & 1.00 & - \\
\hline $348-583$ & $516(22.5 \%)$ & 293(21.8\%) & $0.96(0.78-1.19)$ & 0.746 \\
\hline $584-2778$ & $1112(48.6 \%)$ & $685(51.0 \%)$ & $0.89(0.74-1.07)$ & 0.211 \\
\hline $2779-6944$ & $181(7.9 \%)$ & $109(8.1 \%)$ & $0.91(0.68-1.21)$ & 0.513 \\
\hline$>6944$ & $35(1.5 \%)$ & $13(1.0 \%)$ & $1.47(0.77-2.84)$ & 0.275 \\
\hline \multicolumn{5}{|c|}{ Number of family members (Person) } \\
\hline $1-2$ & $555(24.2 \%)$ & $300(22.3 \%)$ & 1.00 & - \\
\hline $3-5$ & $1401(61.2 \%)$ & $850(63.2 \%)$ & $0.89(0.76-1.05)$ & 0.170 \\
\hline$>5$ & $334(14.6 \%)$ & 194(14.4\%) & 0.93(0.74-1.17) & 0.564 \\
\hline \multicolumn{5}{|c|}{ Average family income (USD) } \\
\hline$<116$ & $495(21.6 \%)$ & $272(20.2 \%)$ & 1.00 & - \\
\hline $116-336$ & $758(33.1 \%)$ & $436(32.4 \%)$ & $0.96(0.79-1.16)$ & 0.665 \\
\hline $337-560$ & $321(14.0 \%)$ & $185(13.8 \%)$ & $0.95(0.76-1.20)$ & 0.720 \\
\hline$>560$ & $716(31.3 \%)$ & $451(33.6 \%)$ & $0.87(0.72-1.05)$ & 0.164 \\
\hline
\end{tabular}

$\mathrm{Cl}$, confidence interval.

Rural, the people who live in rural communities. 
Table 2 Multivariate analysis of demographic characteristics of tuberculosis patients according to Mycobacterium tuberculosis genotype

\begin{tabular}{lcc}
\hline Characteristic & Adjusted ORs (95\% Cl) & $\boldsymbol{P}$ value \\
\hline Age group $<25$ years & $1.35(1.03-1.78)$ & 0.031 \\
\hline Hui Ethnicity & $1.90(1.06-3.41)$ & 0.032 \\
\hline Uyghur Ethnicity & $0.48(0.32-0.72)$ & $<0.001$ \\
\hline Zhuang Ethnicity & $0.34(0.21-0.54)$ & $<0.001$ \\
\hline Urban People & $1.08(0.91-1.28)$ & 0.389 \\
\hline
\end{tabular}

$\mathrm{OR}$, odds ratio; $\mathrm{Cl}$, confidence interval.

[OR (95\% CI): 1.22 (1.03-1.43)] was higher among people infected with Beijing genotype strains than among people infected with non-Beijing genotype strains. We also examined relationship between infection with Beijing genotype strains and clinical symptoms. A cough was the most common symptom for both Beijing and non-Beijing genotype strain infected patients, and there was no significant difference in the incidence of this symptom between these two groups. A significantly lower percentage of Beijing genotype infected patients with hemoptysis [OR (95\% CI): 0.81 (0.69-0.94)] and chest pain [OR (95\% CI): 0.79 (0.69-0.91)] was observed, using patients infected with non-Beijing genotype strains as a reference. In contrast, fever [OR (95\% CI): 1.25 (1.09-1.43)] was more frequently observed in patients infected with Beijing genotype strains than in those infected with non-Beijing genotype strains. In addition, TB contact history and the use of DOTS monitoring did not result in significant differences between the two groups.

\section{Discussion}

To the best of our knowledge, this is the first report of risk factors and clinical phenotypes associated with the Beijing genotype in China. Many of the investigations performed in other countries are limited by sample size or loss of detailed epidemiological information [12,13,24,25]. Using data for 3634 strains identified in the first national drug-resistance surveillance of China, our data describe the detailed population structure of both Beijing and nonBeijing genotype strains.

According to our study, the Beijing genotype is the most predominant genotype in China, and several factors have an influence on its distribution among the

Table 3 Clinical characteristics of tuberculosis patients according to Mycobacterium tuberculosis genotype

\begin{tabular}{|c|c|c|c|c|}
\hline \multirow[t]{3}{*}{ Characteristic } & Beijing genotype & Other genotypes & Odds ratios & $P$ \\
\hline & $(2290)$ & (1344) & $(95 \% \mathrm{Cl})$ & \\
\hline & $\mathrm{N}(\%)$ & $\mathrm{N}(\%)$ & & \\
\hline \multicolumn{5}{|l|}{ Medical history } \\
\hline New case & 1748(76.3\%) & $1071(79.7 \%)$ & 1.00 & - \\
\hline Retreatment case & $542(23.7 \%)$ & $273(20.3 \%)$ & $1.22(1.03-1.43)$ & 0.021 \\
\hline \multicolumn{5}{|c|}{ History of tuberculosis contact } \\
\hline Yes & 1864(81.4\%) & $1081(80.4 \%)$ & 1.00 & - \\
\hline No & $426(18.6 \%)$ & 263(19.6\%) & $0.94(0.79-1.11)$ & 0.483 \\
\hline \multicolumn{5}{|c|}{ Live in area with DOTS implemented } \\
\hline Before 2000 & $1276(55.7 \%)$ & $793(59.0 \%)$ & 1.00 & - \\
\hline 2000 or after & $1014(44.3 \%)$ & $551(41.0 \%)$ & 1.14(1.00-1.31) & 0.056 \\
\hline \multicolumn{5}{|l|}{ Cough } \\
\hline No & $122(5.3 \%)$ & $66(4.9 \%)$ & 1.00 & - \\
\hline$<3$ weeks & $382(16.7 \%)$ & $214(15.9 \%)$ & 0.97(0.69-1.36) & 0.862 \\
\hline$\geq 3$ weeks & $1786(78.0 \%)$ & 1064(79.2\%) & $0.91(0.67-1.24)$ & 0.586 \\
\hline \multicolumn{5}{|l|}{ Hemoptysis } \\
\hline No & $1715(74.9 \%)$ & $949(70.6 \%)$ & 1.00 & - \\
\hline Yes & $575(25.1 \%)$ & $395(29.4 \%)$ & $0.81(0.69-0.94)$ & 0.005 \\
\hline \multicolumn{5}{|l|}{ Fever } \\
\hline No & $1167(51.0 \%)$ & $758(56.4 \%)$ & 1.00 & - \\
\hline Yes & $1123(49.0 \%)$ & $586(43.6 \%)$ & $1.25(1.09-1.43)$ & 0.002 \\
\hline \multicolumn{5}{|l|}{ Chest pain } \\
\hline No & 1549(67.6\%) & $837(62.3 \%)$ & 1.00 & - \\
\hline Yes & $741(32.4 \%)$ & $507(37.7 \%)$ & $0.79(0.69-0.91)$ & 0.001 \\
\hline
\end{tabular}

$\mathrm{OR}$, odds ratio; $\mathrm{Cl}$, confidence interval. 
population. Of all the factors, the correlation between Beijing genotype with MDR and fluoroquinolone resistance might influence the overall risk factors for Beijing spoligotypes compared to other genotypes [21]. In addition, a previous study in Vietnam indicated that the Beijing genotype is associated with young patients (aged less than 25 years), while research performed in other countries has suggested that infection with the Beijing genotype showed no association with a patient's age [13]. Consistent with results from Vietnam, we found that young age (less than 25 years) showed a strong association with infection by the Beijing genotype, suggesting that the Beijing genotype will tend to become dominant in the population in China, possibly because young people tend to engage in greater levels of social interaction. van Soolingen et al. hypothesized that BCG vaccination may be one of the selective forces implicated in the successful spread of the Beijing genotype [26]. This hypothesis is supported by results from other studies [27]. There is speculation that the Beijing genotype strains may have diverged from a common ancestor in the recent past. Although the exact date of their divergence cannot be calculated, it is thought that this evolutionary process has been completed in less than a century [26]. Hence, one possible explanation for the low spread of the Beijing genotype in the older population is that older patients previously infected with the non-Beijing genotype are resistant to the recentlyemerged Beijing genotype strain. Additionally, another possible explanation could be an increased ongoing transmission in the society of this form of $\mathrm{TB}$, which would be reflected in a higher prevalence in the young. Further study is required to confirm the hypothesis.

In addition to age, infection with the Beijing genotype was associated with ethnicity. Compared with Han, people of both Uyghur and Zhuang ethnicities showed a lower than average risk of infection with the Beijing genotype. In a previous study we showed that the percentage of Uyghur patients infected with CAS1-DEHLI genotype strains was significantly higher than that of other ethnicities [21], and suggested that this may be due to the frequent exchange between Uyghur people and ethnicities from several neighboring Central Asian countries where the CAS1-DEHLI genotype is highly prevalent, since they share similar cultural traditions. Since the Zhuang ethnicity is mainly distributed in Guangxi Province in South China, the relatively low incidence of the Beijing genotype is consistent with the relatively low percentage of Beijing genotype strains in Southern China [21]. In contrast to other ethnic minorities, Hui ethnicity is defined rather based on cultural or religious reasons instead of genetic one. Hence, the special traditions and ways of living in Hui ethnicity may be responsible for the high percentage of Beijing genotype.
However, a larger study is needed to answer additional questions about the distribution of Beijing genotype and non-Beijing genotype strains among different ethnicities.

With respect to occupation, we found strong associations between urban people and infection with Beijing genotype strains. In China, rural people are usually living in rural area with less density of population, who depend on argricultural activities for their livelihoods, and therefore their mainly work condition is outside the building; whereas urban people tend to live in urban areas with more density of population and always work inside the crowd public environment, which contribute to Beijing infection of urban people. In addition, many studies have suggested that the Beijing genotype is associated with resistance to anti-tuberculosis drugs [18,28-30]. Due to the unbalanced distribution of health care resources in China [31], misuse and overuse of antibiotics is more common in urban areas than in rural areas in China, and this may account for the higher prevalence of drugresistant Beijing genotype strains among urban people.

In addition to demographic characteristics, patients infected with Beijing genotype strains presented different clinical profiles compared with those infected with nonBeijing genotype strains. Both hemoptysis and chest pain are common manifestations of pulmonary tuberculosis [32]; moreover, massive hemoptysis is a life-threatening condition with a poor treatment outcome in active tuberculosis patients [33]. In this study, we observed that $25.1 \%$ and $32.4 \%$ of patients infected with Beijing strains had hemoptysis and chest pain, respectively, compared with $29.4 \%$ and $37.7 \%$, respectively, of those infected with non-Beijing strains. These differences were statistically significant. That both symptoms have a lower occurrence in patients infected with the Beijing strain is reasonable as the two symptoms often occur together in the same patient. Our data indicate that chest symptoms occur more frequently in patients infected with nonBeijing strains. Our results are in agreement with a study performed in Singapore which demonstrated that there is a significantly higher frequency of cavitary disease in patients infected with non-Beijing genotype strains [25]. Nevertheless, in studies on the CXR presentation of patients completed in Indonesia and in the Netherlands $[12,20]$, the authors did not find any significant difference between the two groups. While more attention has been given to the Beijing genotype than to other genotypes due to the association of its supposed higher virulence with relapse and treatment failure in humans [13,20,34-36], our findings suggest that patients infected with non-Beijing genotype strains may have more serious chest phenotypes.

Fever is a typical clinical symptom of tuberculosis patients, and recent findings have revealed that the frequency with which fever is observed is more frequently 
reported in non-Beijing genotype infected patients than in patients infected with the Beijing genotype [25]. In contrast, we found that fevers occurred in a significantly higher proportion of patients infected with Beijing genotype strains in China than in those infected with nonBeijing genotype strains. Our results are in agreement with a cohort study by Parwati et al. which showed that more patients infected with Beijing genotype strains developed fever during treatment [20].

\section{Conclusion}

In summary, we have shown that ethnicity, occupation and earlier treated patients are risk factors for infection with Beijing genotype strains, and identified clinical phenotypes that are strongly associated with the genotype of the strain with which a patient is infected. Our data suggest that urban people, Hui ethnicity and earlier treated patients are all high risk factors, while older age group, and Uyghur or Zhuang ethnicity show a lower than average risk for infection with the Beijing genotype. Other characteristics, including sex, economic situation, contact history and implementation of DOTS monitoring did not vary between patients infected with Beijing and non-Beijing genotype strains. In addition, the high proportion of hemoptysis and chest pain appearing in nonBeijing genotype infected patients indicates that more attention should be given to basic research on non-Beijing genotype strains.

\section{Competing interests}

The authors declare that they have no competing interests.

\section{Authors' contributions}

Conceived and designed the experiments: YLZ YP HX YYS. Performed the experiments: YP YYS HX YZ BZ. Analyzed the data: YP HX. Wrote the paper: YLZ YP. All authors read and approved the final manuscript.

\section{Acknowledgments}

We are grateful to Dr. Joy Fleming for helpful discussions and for improving the English of this manuscript, and to all the members of the National Tuberculosis Reference Laboratory at the Chinese Center for Disease Control and Prevention for their cooperation and technical help. We also thank the reviewers for helpful comments and critiques. This study was supported by the National Key Research Program of China (2008ZX10003-009).

Received: 29 November 2011 Accepted: 19 November 2012 Published: 17 December 2012

\section{References}

1. Walls T, Shingadia D: The epidemiology of tuberculosis in Europe. Arch Dis Child 2007, 92:726-729.

2. Lari N, Rindi L, Bonanni D, Rastogi N, Sola C, Tortoli E, Garzelli C: Three-year longitudinal study of genotypes of mycobacterium tuberculosis isolates in Tuscany, Italy. J Clin Microbiol 2007, 45:1851-1857.

3. World Health Organization: Global tuberculosis control report. 2010, http://www.who.int/tb/publications/global_report/2010/en/.

4. Wang Y: Report of 5th national TB epidemiological survey in China. Beijing: Military Medical Science Press; 2011.

5. van Soolingen D, Qian L, de Haas PE, Douglas JT, Traore H, Portaels F, Qing HZ, Enkhsaikan D, Nymadawa P, van Embden JD: Predominance of a single genotype of mycobacterium tuberculosis in countries of east Asia. J Clin Microbiol 1995, 33(12):3234-3238.
6. Bifani PJ, Mathema B, Kurepina NE, Kreiswirth BN: Global dissemination of the mycobacterium tuberculosis W-Beijing family strains. Trends Microbiol 2002, 10:45-52.

7. Glynn JR, Whiteley J, Bifani PJ, Kremer K, van Soolingen D: Worldwide occurrence of Beijing/W strains of mycobacterium tuberculosis: a systematic review. Emerg Infect Dis 2002, 8:843-849.

8. Mokrousov I, Jiao WW, Sun GZ, Liu JW, Valcheva V, Li M, Narvskaya O, Shen AD: Evolution of drug resistance in different sublineages of mycobacterium tuberculosis Beijing genotype. Antimicrob Agents Chemother 2006, 50:2820-2823.

9. Dong H, Liu Z, Lv B, Zhang Y, Liu J, Zhao X, Wan K: Spoligotypes of mycobacterium tuberculosis from different provinces of china. J Clin Microbiol 2010, 48(11):4102-4106.

10. Kruuner A, Hoffner SE, Sillastu H, Danilovits M, Levina K, Svenson SB, Ghebremichael S, Koivula T, Kallenius G: Spread of drug-resistant pulmonary tuberculosis in Estonia. J Clin Microbiol 2001, 39:3339-3345.

11. Caminero JA, Pena MJ, Campos-Herrero MI, Rodriguez JC, Garcia I, Cabrera P, Lafoz C, Samper S, Takiff H, Afonso O, et al: Epidemiological evidence of the spread of a mycobacterium tuberculosis strain of the Beijing genotype on Gran canaria island. Am J Respir Crit Care Med 2001, 164:1165-1170.

12. Borgdorff MW, de Haas P, Kremer K, van Soolingen D: Mycobacterium tuberculosis Beijing genotype, the Netherlands. Emerg Infect Dis 2003, 9:1310-1313.

13. Anh DD, Borgdorff MW, Van LN, Lan NT, van Gorkom T, Kremer K, van Soolingen D: Mycobacterium tuberculosis Beijing genotype emerging in Vietnam. Emerg Infect Dis 2000, 6:302-305.

14. Lan NT, Lien HT, Tung le B, Borgdorff MW, Kremer K, van Soolingen D: Mycobacterium tuberculosis Beijing genotype and risk for treatment failure and relapse, Vietnam. Emerg Infect Dis 2003, 9:1633-1635.

15. Drobniewski F, Balabanova Y, Nikolayevsky V, Ruddy M, Kuznetzov S, Zakharova S, Melentyev A, Fedorin I: Drug-resistant tuberculosis, clinical virulence, and the dominance of the Beijing strain family in Russia. JAMA 2005, 293:2726-2731.

16. European Concerted Action on New Generation Genetic Markers and Techniques for the Epidemiology and Control of Tuberculosis: Beijing/W genotype mycobacterium tuberculosis and drug resistance. Emerg Infect Dis 2006, 12:736-743.

17. Lasunskaia E, Ribeiro SC, Manicheva O, Gomes LL, Suffys PN, Mokrousov I, Ferrazoli L, Andrade MR, Kritski A, Otten T, et al: Emerging multidrug resistant mycobacterium tuberculosis strains of the Beijing genotype circulating in Russia express a pattern of biological properties associated with enhanced virulence. Microbes Infect 2010, 12(6):467-475.

18. Buu TN, Huyen MN, Lan NT, Quy HT, Hen NV, Zignol M, Borgdorff MW, Cobelens FG, van Soolingen D: The Beijing genotype is associated with young age and multidrug-resistant tuberculosis in rural Vietnam. Int $J$ Tuberc Lung Dis 2009, 13:900-906.

19. Caws M, Thwaites G, Stepniewska K, Nguyen TN, Nguyen TH, Nguyen TP, Mai NT, Phan MD, Tran HL, Tran TH, et al: Beijing genotype of mycobacterium tuberculosis is significantly associated with human immunodeficiency virus infection and multidrug resistance in cases of tuberculous meningitis. J Clin Microbiol 2006, 44:3934-3939.

20. Parwati I, Alisjahbana B, Apriani L, Soetikno RD, Ottenhoff TH, van der Zanden AG, van der Meer J, van Soolingen D, van Crevel R: Mycobacterium tuberculosis Beijing genotype is an independent risk factor for tuberculosis treatment failure in Indonesia. J Infect Dis 2010, 201:553-557.

21. Pang Y, Zhou Y, Zhao B, Liu G, Jiang G, Xia H, Song Y, Shang Y, Wang S, Zhao YL: Spoligotyping and drug resistance analysis of mycobacterium tuberculosis strains from national survey in China. PLoS One 2012, 7(3):e32976.

22. Zhao $Y$, Xu S, Wang L, Chin DP, Wang S, Jiang G, Xia H, Zhou Y, Li Q, Ou X, et al: National survey of drug-resistant tuberculosis in China. N Engl J Med 2012, 366(23):2161-2170.

23. Pang Y, Zhou Y, Wang S, Lu J, Lu B, He G, Wang L, Zhao Y: A novel method based on high resolution melting (HRM) analysis for MIRU-VNTR genotyping of mycobacterium tuberculosis. J Microbiol Methods 2011, 86:291-297.

24. Feng JY, Su WJ, Tsai CC, Chang SC: Clinical impact of mycobacterium tuberculosis W-Beijing genotype strain infection on aged patients in Taiwan. J Clin Microbiol 2008, 46:3127-3129. 
25. Sun YJ, Lim TK, Ong AK, Ho BC, Seah GT, Paton NI: Tuberculosis associated with mycobacterium tuberculosis Beijing and non-Beijing genotypes: a clinical and immunological comparison. BMC Infect Dis 2006, 6:105.

26. Parwati I, van Crevel R, van Soolingen D: Possible underlying mechanisms for successful emergence of the mycobacterium tuberculosis Beijing genotype strains. Lancet Infect Dis 2010, 10:103-111.

27. Lopez B, Aguilar D, Orozco H, Burger M, Espitia C, Ritacco V, Barrera L, Kremer K, Hernandez-Pando R, Huygen K, van Soolingen D: A marked difference in pathogenesis and immune response induced by different mycobacterium tuberculosis genotypes. Clin Exp Immunol 2003, 133:30-37.

28. Bifani PJ, Plikaytis BB, Kapur V, Stockbauer K, Pan X, Lutfey ML, Moghazeh SL, Eisner W, Daniel TM, Kaplan MH, et al: Origin and interstate spread of a New York city multidrug-resistant mycobacterium tuberculosis clone family. JAMA 1996, 275:452-457.

29. Balabanova la M, Nikolaevskii W, Raddi M, Drobnevskii F, Fedorin IM, Erokhin W, Kuznetsov SI, Zakharova SM, Melent'ev AS, Chernousova LN, Golyshevskaia Vl: Preponderance of mycobacterium tuberculosis strains of the family Beijing and risk factors of their transmission in the samara region. Probl Tuberk Bolezn Legk 2006, 9:31-37.

30. Almeida D, Rodrigues C, Ashavaid TF, Lalvani A, Udwadia ZF, Mehta A: High incidence of the Beijing genotype among multidrug-resistant isolates of mycobacterium tuberculosis in a tertiary care center in Mumbai India. Clin Infect Dis 2005, 40:881-886

31. Gao J, Qian J, Tang S, Eriksson BO, Blas E: Health equity in transition from planned to market economy in China. Health Policy Plan 2002, 17(Suppl):20-29.

32. Leung AN: Pulmonary tuberculosis: the essentials. Radiology 1999, 210:307-322.

33. Muthuswamy PP, Akbik F, Franklin C, Spigos D, Barker WL: Management of major or massive hemoptysis in active pulmonary tuberculosis by bronchial arterial embolization. Chest 1987, 92:77-82.

34. Abebe F, Bjune $\mathrm{G}$ : The emergence of Beijing family genotypes of Mycobacterium tuberculosis and low-level protection by bacille Calmette-Guerin (BCG) vaccines: is there a link? Clin Exp Immunol 2006, 145:389-397.

35. Fortes A, Pereira K, Antas PR, Franken CL, Dalcolmo M, Ribeiro-Carvalho MM, Cunha KS, Geluk A, Kritski A, Kolk A, et al: Detection of in vitro interferongamma and serum tumour necrosis factor-alpha in multidrug-resistant tuberculosis patients. Clin Exp Immunol 2005, 141:541-548.

36. Manca C, Reed MB, Freeman S, Mathema B, Kreiswirth B, Barry CE III, Kaplan G: Differential monocyte activation underlies strain-specific mycobacterium tuberculosis pathogenesis. Infect Immun 2004, 72:5511-5514.

doi:10.1186/1471-2334-12-354

Cite this article as: Pang et al:: Risk factors and clinical phenotypes of Beijing genotype strains in tuberculosis patients in China. BMC Infectious Diseases 2012 12:354.

\section{Submit your next manuscript to BioMed Central and take full advantage of:}

- Convenient online submission

- Thorough peer review

- No space constraints or color figure charges

- Immediate publication on acceptance

- Inclusion in PubMed, CAS, Scopus and Google Scholar

- Research which is freely available for redistribution

Submit your manuscript at www.biomedcentral.com/submit
C BioMed Central 